\title{
Elderliness and Obesity
}

\section{İbrahim Halil Türkbeyler ${ }^{*}$, Eyyüp Murat Efendioğlu², Ahmet Çiğiloğlu², Azer Abiyev $^{3}$, Zeynel Abidin Öztürk ${ }^{2}$}

\begin{abstract}
${ }^{1}$ Ersin Arslan Education and Research Hospital, Department of Internal Medicine, Division of Geriatrics, Gaziantep, Turkey.

${ }^{2}$ Gaziantep University, Faculty of Medicine, Department of Internal Medicine, Division of Geriatrics, Gaziantep, Turkey.

${ }^{3}$ Gaziantep University, Faculty of Medicine, Department of Internal Medicine, Gaziantep, Turkey.
\end{abstract}

\begin{abstract}
World becomes more and more elderly; on the other hand, obesity is on the rise. The frequency of obesity is exceedingly high in geriatric individuals and it is associated with many diseases as well as leading to a decrease in quality of life and disability in the elderly. The aim of this study is to compare Comprehensive Geriatric Assessment of geriatric obese and non-obese patients. 39 normal weight and 41 obese patients who applied to Geriatric Clinic for various reasons were included in the study. Basic activities of daily Life, instrumental activities of daily living, Tinetti balance and gait assessment, time up and go, mini mental state examination, mini nutritional assessment, Yesavage geriatric depression and Pittsburgh sleep quality indices tests were applied to the patients and also gait speed and hand grip strength of each patient was determined. Consequently, obese elderly patients were found to be deteriorated in some physical abilities and functions which are needed to maintain daily life such as gait and balance, muscle strength, certain daily life activities and mood and sleep quality compared to normal weight.
\end{abstract}

Key words: Elderliness, Obesity, Life quality.

\section{Introduction}

Elderly period of the life with its all difficulties is the most challenging issue facing the medicine. As many regressions in all physiological systems are encountered during this period, the most correct advices should be provided for elderly individuals and the best treatment approaches should be exhibited. Obesity can cause morbidity, functional disability and even early deaths as a global problem that can be seen in all age groups, in all developed and developing countries. Obesity is already defined as excess body fat which might result in many diseases and even premature death of individual. Obesity is related to several diseases including diabetes mellitus, hypertension, dyslipidaemia, coronary

\footnotetext{
* Corresponding author: İbrahim Halil Türkbeyler, Tel: +90 505488 2185, E-mail: ihturkbeyler@gmail.com, ORCID ID: 0000-0002-5489-1692.
} 
artery disease, heart disease and stroke, osteoarthritis, breathing problems such as sleep apnoea and some cancers. It is very well known that the risk of death because of all causes increases as an individual moves towards peak of the range of moderate to severe obesity (1). Besides, it reduces life expectancy, obesity decreases the number of years to be lived without cardiovascular diseases (2). Approximately, $32 \%$ of adults in USA are obese, $20 \%$ of them are obese in Canada and it is $30.5 \%$ in Turkey (3-5). The prevalence of obesity has been steadily increasing among older age group in both developed and developing countries. $39.3 \%$ of individuals aged 65 and above are displaying abdominal obesity in Turkey and nearly $80 \%$ percent of them have a waist to hip ratio beyond acceptable range determined by World Health Organization (WHO) (6).

The aim of this study is to comprehensively compare the obese and non - obese elderly individuals by using certain assessment tools which are uniquely applied in outpatient clinics, hospitals, and nursing homes. Older adults are already under the effect of many medical, psychiatric, or social troubles. Most probably, obesity furnishes elderly individuals with some additional adverse outcomes independent of already present medical, psychiatric, or social circumstances.

\section{Method}

39 normal weight and 41 obese male elderly patients who applied to geriatric outpatient clinics for various reasons were included in the study. The study with a research protocol number of 296 was approved by the Gaziantep University Local Research Ethics Committee. Basic activities of daily life (BADLs), instrumental activities of daily living (IADLs), Tinetti balance and gait assessment, time up and go, mini mental state examination, mini nutritional assessment, Yesavage geriatric depression and Pittsburgh sleep quality indices tests were applied to the patients and also gait speed and hand grip strength of each patient was determined. Whether the descriptive statistical values in two groups are normally distributed or not was revealed before each comparison. All descriptive statistics were found to be normally distributed and expressed as mean \pm standard deviation. Student $t$ test was used for comparisons of normally distributed descriptive values. Two sided values of $\mathrm{p}<0.05$ were considered as statistically significant.

\section{Results}

There was no statistically significant difference between two groups in terms of age and gender. Basic activities of daily life (BADLs), mini mental state examination (MMSE) and mini nutritional assessment test scores also displayed no significance. Waist circumferences, hip circumference, mid - upper arm circumference and body mass index scores were noteworthily higher in obese group, as it is readily expected. Not surprisingly, non - obese group had better scores in other assessment tests (Table 1). 
Table 1: General features and results of tests $(\mathrm{p}<0.05$ is accepted as statistically different)

\begin{tabular}{lccc}
\hline & Non - obese $(\mathbf{n}=\mathbf{3 9})$ & Obese $(\mathbf{n}=\mathbf{4 1})$ & $\mathbf{p}$ \\
\hline Age & $71.18 \pm 6.02$ & $71.54 \pm 5.80$ & $\mathrm{p}>0.05$ \\
Gender (M/F) & $20 / 19$ & $21 / 20$ & $\mathrm{p}>0.05$ \\
Waist Circumference (cm) & $97.57 \pm 9.84$ & $108.92 \pm 8.60$ & $\mathrm{p}=0.001$ \\
Hip Circumference (cm) & $105.32 \pm 7.35$ & $118.65 \pm 9.60$ & $\mathrm{p}=0.001$ \\
Mid - Upper Arm Circumference (cm) & $29.57 \pm 2.31$ & $33.00 \pm 3.30$ & $\mathrm{p}=0.001$ \\
Body Mass Index (kg/m $)^{2}$ & $26.11 \pm 2.59$ & $33.49 \pm 3.42$ & $\mathrm{p}=0.001$ \\
Gait Speed (m/s) & $0.88 \pm 0.37$ & $0.62 \pm 0.33$ & $\mathrm{p}=0.002$ \\
Hand Grip Strength (kg) & $26.18 \pm 10.45$ & $18.26 \pm 10.43$ & $\mathrm{p}=0.001$ \\
Basic Activities of Daily Life & $17.62 \pm 4.50$ & $21.58 \pm 5.22$ & $\mathrm{p}>0.05$ \\
Instrumental Activities of Daily Living & $6.42 \pm 1.26$ & $5.68 \pm 1.36$ & $\mathrm{p}=0.015$ \\
Tinetti Balance and Gait Assessment & $23.18 \pm 4.15$ & $20.43 \pm 4.41$ & $\mathrm{p}=0.006$ \\
Time Up and Go (s) & $10.35 \pm 4.83$ & $13.24 \pm 5.28$ & $\mathrm{p}=0.014$ \\
Mini Mental State Examination & $24.84 \pm 4.73$ & $24.80 \pm 4.51$ & $\mathrm{p}>0.05$ \\
Yesavage Geriatric Depression Scale & $2.89 \pm 0.51$ & $4.68 \pm 0.65$ & $\mathrm{p}=0.035$ \\
Mini Nutritional Assessment & $11.42 \pm 2.45$ & $11.05 \pm 2.75$ & $\mathrm{p}>0.05$ \\
Pittsburgh Sleep Quality Index & $4.74 \pm 2.35$ & $7.24 \pm 3.92$ & $\mathrm{p}=0.001$ \\
\hline
\end{tabular}

\section{Discussion}

There are many kinds of Comprehensive Geriatric Assessment tools each of which is prepared for different healthcare settings and to meet different needs, eventually to manage frail geriatric patients. In our study, obese and non - obese groups did not display any significance in terms of age and gender. But there were different scores worth mentioning here between groups when we use certain tools to assess geriatric patients.

First, there was no difference between BADLs scores. However, BADLs tool is used to assess the most basic daily activities such as bathing, dressing, maintaining continence, toileting, grooming, feeding and transferring. However, mainly instrumental activities of daily living (IADLs) scores of individuals should be examined to consider someone has the ability of maintaining an independent household or not. Someone might be adequate in BADLs tool but cannot live independently in community. Mental goodness of individual is demonstrated by using IADLs. Individuals with mild cognitive impairment (MCI) who have impairments performing IADLs are more likely to develop dementia (7). Obese geriatric patients had poor IADLs scores in our study. In our study, non - obese patients could not have a mean score above 24 . But obese patients have significantly lower scores than the non - obese patient.

The autonomy and independence are substantial for every person. One of the most basic approaches to evaluate independence and physical frailty of an elderly is undoubtedly measurement of gait speed. The mean gait speed of non - obese elderly individuals in our study was comparable to previous studies (8). The gait speed of obese group was significantly lower than non - obese group. Some scientists propose grip strength as a biomarker of present health status of an elderly because there is a strong association between hand grip strength and strength of other muscles in the body and how 
influential the muscle actions of an elderly individual (9). As it could be expected, hand grip strength of obese elderly individuals was significantly lower in our study.

Fall prevention is also a substantial issue for management of geriatric patients in clinical practice. Tinetti balance and gait assessment tool provides the most precious fall risk evaluation in the elderly $(10,11)$. According to this test tool, individuals score 24 and above are considered to have a low fall risk. Those of individuals score between 19 and 23 have a moderate risk and individuals score 18 or lower have a high fall risk.

One of the other important testing tools to evaluate the fall risk of an individual is the time up and go test. Indeed, observation of gait quality of an elderly is a suggestive examination method in clinical practice. Most of the time, gait assessment is ignored in physical examination by practitioners, even when fall is a one of major complaints of a patient. The time up and go test consists of certain period evaluations. How much time an elderly individual needs to get up from a chair, walk 3 meters, turn back, and sit on the chair is inquired during the TUG test. Time less than $15 \mathrm{~s}$ is considered a plausibly low fall risk for that patient (12, 13). In our study, both the obese and non obese groups had better mean values than 15 s. Indeed, non - obese group was significantly much better than obese group. Assessment of mental status, especially in patients with neurobehavioral disorders, provides valuable clues for both diagnosing and treatment. This assessment should be certainly following physical and neurological examination. The MMSE truly tests episodic memory and orientation of the patient. According to MMSE scores, there was no significant difference between groups, in our study. Mini mental state examination test is frequently used screening test for MCI diagnosis (14). 20 $22 \%$ of 71 year olds were reported as mild cognitive impairment (MCI) \& cognitive impairment, no dementia, in the United States (15). Despite the fact that, mid-life overweight or obesity is a prominent risk factor prompting Alzheimer's disease (AD) or vascular type of dementia, some studies propose no difference between cognitive functions of obese and non - obese people (16). Body mass index is alone a predictor of temporal lobe atrophy (17). Obesity, independent of any other risk factor, is known to increase the risk of MCI $(18,19)$. It doubles the risk of $\mathrm{AD}$ and mid-life obesity absolutely increase the risk of dementia in later life (20). However, there are also some studies displaying no association between obesity and dementia. In a recent retrospective cohort study, finding precisely contradicts the hypothesis that the mid-life obesity is proposed to lead to dementia in later life (21).

Besides dementia, depression is also known to be prevalent among elderly individuals. Depression among the elderly aged 65 and above, with no previous history of depression is named as late-onset depression. With a progressively increasing old age population worldwide, diagnosing depression in older adults is extremely important, because they may display different symptoms than middle-aged individuals and need diverse treatment modalities. Also an association between obesity and depression has been frequently established (22). It is often proposed that social isolation due to stigmatization of obese individuals can prompt depression. It is found that increases in BMI result in increased depression days or days having had while an individual is being depressed 
(23). According to our study, obese elderly adults were within the mild depression range of Yesavage depression scale, whereas non - obese elderly adults were in normal range.

The mini nutritional assessment is an uncomplicated and extremely sensitive tool for nutritional screening and assessment (24). It particularly provides rapid assessment of nutritional status of the elderly in outpatient and inpatients clinics and nursing homes. With this test, elderly individuals who are in malnutrition or at risk of malnutrition can be determined. All participants in our study were below the range accepted as normal nutritional status. Mean of both groups were just below 12 points which is accepted as lower border of the normal range and there was no significant difference between groups.

It is also very well known that subjective sleep quality deteriorates with aging. Certain medical and psychiatric problems may contribute to that deterioration, too $(25,26)$. Obese elderly individuals in our study had obviously a poor sleep quality in comparison to non - obese ones.

In our study, obese patients are obviously found to have a lower performance than non - obese ones in gait speed, hand grip strength, instrumental activities of daily living, Tinetti balance and gait assessment, Time up and go, Yesavage geriatric depression scale, and Pittsburgh sleep quality tests. The relationship between obesity and mortality and whether we should advice patient to lose weight in elderly period, have still carried on remaining controversial. There are studies implicating a $\mathrm{U}$ - shaped relationship between obesity and mortality in elderly. It is mostly found that obesity rather than overweight results in a moderately high risk of mortality in elderly period. Also, threshold value at which BMI confers increased mortality risk to the elderly is found to be higher than that of younger adults. This is considered as "obesity paradox" (27-29). In our study, it seems that the obesity readily reduces quality of life.

\section{Conclusion:}

At the end of a comprehensive geriatric assessment, some pathfinder findings which can be arousal for a practitioner who examine an obese elderly were obtained. Obesity results in some additional consequences which certainly decrease the quality of life in the elderly other than outcomes of any medical and psychiatric circumstances.

\section{Conflict of interest}

The authors declare that no conflict of interest exists.

\section{Acknowledgement}

No institution has given financial support to the study. All researchers contributed equally to the study.

\section{References}

1. Calle EE, Thun MJ, Petrelli JM, et al. Body-mass index and mortality in a prospective cohort of U.S. adults. The New England journal of medicine. 1999;341(15):1097-105.

2. Pardo Silva MC, De Laet C, Nusselder WJ, et al. Adult obesity and number of years lived with and without cardiovascular disease. Obesity. 2006;14(7):1264-73.

3. Ogden CL, Carroll MD, Curtin LR, et al. Prevalence of overweight and obesity in the United States, 1999-2004. Jama. 2006;295(13):1549-55.

4. Sokar-Todd HB, Sharma AM. Obesity research in Canada: literature overview of the last 3 decades. Obes Res. 2004;12(10):1547-53.

5. Ardahan M, Konal E. The prevalence of hypertension and obesity and effective factors: A cross-sectional bazaar study. JPMA The Journal of the Pakistan Medical Association. 2019;69(7):101821. 
6. Pekcan AG, Samur G, Dikmen D, et al. Population based study of obesity in Turkey: results of the Turkey Nutrition and Health Survey(TNHS)2010. Progress in Nutrition. 2017;19(3):248-56.

7. Guo HJ, Sapra A. Instrumental Activity of Daily Living (IADL). StatPearls. Treasure Island (FL)2020.

8. Busch Tde A, Duarte YA, Pires Nunes D, et al. Factors associated with lower gait speed among the elderly living in a developing country: a cross-sectional population-based study. BMC geriatrics. 2015;15:35.

9. Bohannon RW. Grip Strength: An Indispensable Biomarker For Older Adults. Clinical interventions in aging. 2019;14:1681-91.

10. Perell KL, Nelson A, Goldman RL, et al. Fall risk assessment measures: an analytic review. The journals of gerontology Series A, Biological sciences and medical sciences. 2001;56(12):M7616.

11. Kopke S, Meyer G. The Tinetti test: Babylon in geriatric assessment. Zeitschrift fur Gerontologie und Geriatrie. 2006;39(4):288-91.

12. Browne W, Nair BKR. The Timed Up and Go test. The Medical journal of Australia. 2019;210(1):13-4 e1.

13. Podsiadlo D, Richardson S. The timed "Up \& Go": a test of basic functional mobility for frail elderly persons. Journal of the American Geriatrics Society. 1991;39(2):142-8.

14. Onwuekwe I. Assessment of mild cognitive impairment with mini mental state examination among adults in southeast Nigeria. Annals of medical and health sciences research. 2012;2(2):99102.

15. Plassman BL, Langa KM, Fisher GG, etal. Prevalence of cognitive impairment without dementia in the United States. Annals of internal medicine. 2008;148(6):427-34.

16. Conforto RM, Gershman L. Cognitive processing differences between obese and nonobese subjects. Addictive behaviors. 1985;10(1):83-5.

17. Gustafson D, Lissner L, Bengtsson C, et al. A 24-year follow-up of body mass index and cerebral atrophy. Neurology. 2004;63(10):1876-81. 18. Hassing LB, Dahl AK, Pedersen NL, et al. Overweight in midlife is related to lower cognitive function 30 years later: a prospective study with longitudinal assessments. Dementia and geriatric cognitive disorders. 2010;29(6):543-52.
19. Dahl A, Hassing LB, Fransson E, et al. Being overweight in midlife is associated with lower cognitive ability and steeper cognitive decline in late life. The journals of gerontology Series A, Biological sciences and medical sciences. 2010;65(1):57-62.

20. Anstey KJ, Cherbuin N, Budge M, et al. Body mass index in midlife and late-life as a risk factor for dementia: a meta-analysis of prospective studies. Obesity reviews : an official journal of the International Association for the Study of Obesity. 2011;12(5):e426-37.

21. Qizilbash N, Gregson J, Johnson ME, etal. $\mathrm{BMI}$ and risk of dementia in two million people over two decades: a retrospective cohort study. The lancet Diabetes \& endocrinology. 2015;3(6):431-6.

22. Luppino FS, de Wit LM, Bouvy PF, et al. Overweight, obesity, and depression: a systematic review and meta-analysis of longitudinal studies. Archives of general psychiatry. 2010;67(3):220-9.

23. Ha H, Han C, Kim B. Can Obesity Cause Depression? A Pseudo-panel Analysis. Journal of preventive medicine and public health $=$ Yebang Uihakhoe chi. 2017;50(4):262-7.

24. Guigoz Y, Vellas B. The Mini Nutritional Assessment (MNA) for grading the nutritional state of elderly patients: presentation of the MNA, history and validation. Nestle Nutrition workshop series Clinical \& performance programme. 1999;1:3-11; discussion -2 .

25. Buysse DJ, Reynolds CF, 3rd, Monk TH, et al. The Pittsburgh Sleep Quality Index: a new instrument for psychiatric practice and research. Psychiatry research. 1989;28(2):193-213.

26. Wang P, Song L, Wang K, et al. Prevalence and associated factors of poor sleep quality among Chinese older adults living in a rural area: a population-based study. Aging Clin Exp Res. 2020;32(1):125-31.

27. Mathus-Vliegen EM. Obesity and the elderly. Journal of clinical gastroenterology. 2012;46(7):533-44.

28. Janssen I, Mark AE. Elevated body mass index and mortality risk in the elderly. Obesity reviews : an official journal of the International Association for the Study of Obesity. 2007;8(1):4159.

29. Chapman IM. Obesity paradox during aging. Interdisciplinary topics in gerontology. 2010;37:20-36 